PROFESIONALES Y HERRAMIENTAS PARA EL DESARROLLO LOCAL Y SUS SINERGIAS TERRITORIALES. EVALUACIÓN Y PROPUESTAS DE FUTURO IX Coloquio Nacional de Desarrollo Local del GTDL-AGE 

ANTONIO MARTÍNEZ PUCHE, XAVIER AMAT MONTESINOS, ISABEL SANCHO CARBONELL y DANIEL SANCHIZ CASTAÑO (EDS.)

\section{PROFESIONALES Y HERRAMIENTAS PARA EL DESARROLLO LOCAL Y SUS SINERGIAS TERRITORIALES. EVALUACIÓN Y PROPUESTAS DE FUTURO}

IX Coloquio Nacional de Desarrollo Local del GTDL-AGE

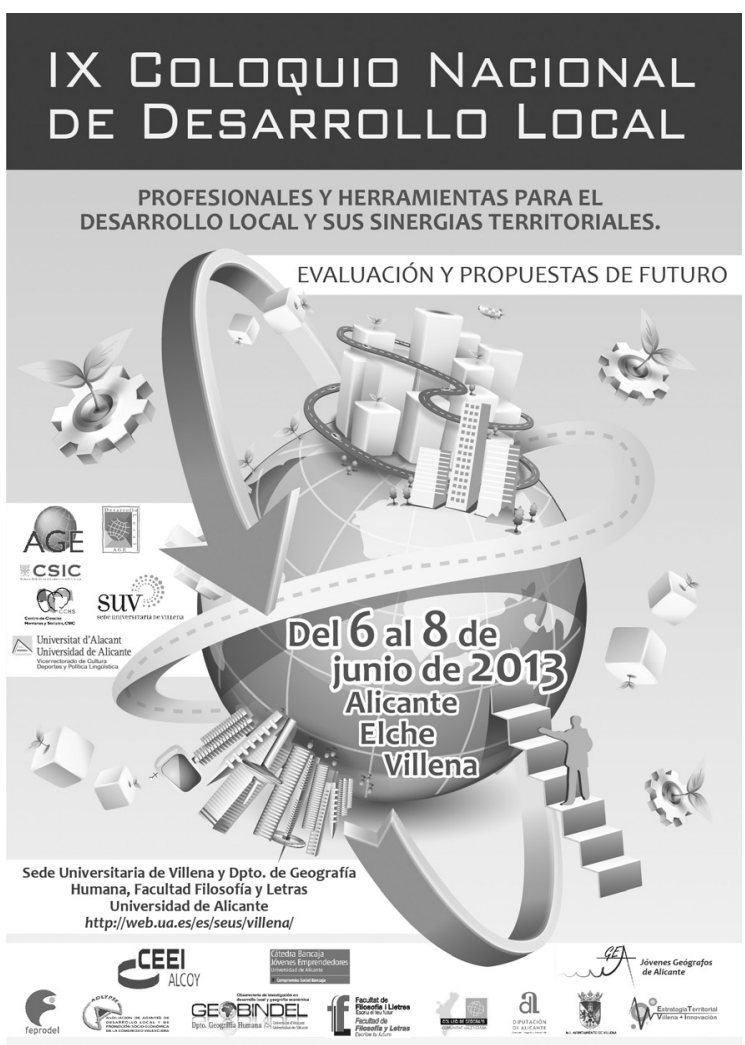


Este libro ha sido examinado y valorado por evaluadores ajenos a la Universidad de Alicante, con el fin de garantizar la calidad científica del mismo.

Publicacions de la Universitat d'Alacant

03690 Sant Vicent del Raspeig

Publicaciones@ua.es

http://publicaciones.ua.es

Telèfon: 965903480

(C) Antonio Martínez Puche, Xavier Amat Montesinos,

Isabel Sancho Carbonell y Daniel Sanchiz Castaño (eds.), 2016

(C) d'aquesta edició: Universitat d'Alacant

ISBN: 978-84-16724-00-0

Dipòsit legal: A 92-2016

Disseny de coberta: candela ink

Composició: Página Maestra (Miguel Ángel Sánchez Hernández)

Impressió i enquadernació: Guada Impresores

\section{unte \\ Unión de Editoriales
Universitarias Españolas \\ WWW.une.es
WWA}

Esta editorial es miembro de la UNE, cosa que garantiza la difusión y comercialización nacional y internacional de sus publicaciones.

Reservados todos los derechos. Cualquier forma de reproducción, distribución, comunicación pública o transformación de esta obra sólo puede ser realizada con la autorización de sus titulares, salvo excepción prevista por la ley. Diríjase a CEDRO (Centro Español de Derechos Repográficos, www.cedro.org) si necesita fotocopias o escanear algún fragmento de esta obra. 


\title{
LA PARTICIPACIÓN CIUDADANA COMO HERRAMIENTA PARA LA GESTIÓN DE LA BIODIVERSIDAD VEGETAL EN LA SERRA DE MARIOLA
}

\author{
Joaquín Palací Soler \\ Crea360 Consultoría de Desarrollo Local \\ joaquin.palaci@geografos.org \\ Antonio Belda Antolí, \\ Dpto. Ciencias de la Tierra y Medio Ambiente \\ Universidad de Alicante \\ antonio.belda@ua.es
}

RESUMEN

La Sierra de Mariola es un parque natural con gran diversidad de flora útil y con un elevado valor etnobotánico, prueba de ello son la existencia de numerosos estudios y referencias bibliográficas vinculadas a este ámbito territorial realizadas en los dos últimos siglos. Teniendo en cuenta la riqueza florística de la zona y la relación existente entre el espacio natural y la población del ámbito de estudio, hemos entendido que la participación ciudadana constituye una herramienta fundamental para la gestión, conservación y la puesta en valor de la flora con valores terapéuticos en este espacio natural protegido.

Con este estudio pretendemos poner en relieve la importancia de los conocimientos que ha adquirido, a lo largo de la historia, la sociedad que vive en el entorno de Mariola. El proceso de investigación se ha hecho efectivo mediante la observación participativa, basado en la consulta individualizada a personas conocedoras de las especies florísticas más representativas del entorno. Toda la información obtenida fruto de los procesos de participación ciudadana ha sido canalizada, gestionada y organizada mediante la utilización de Sistemas de Información Geográfica (SIG), consiguiendo de este modo un inventario florístico dinámico y constituido de las especies más representativas con propiedades medicinales en Mariola. Esta información ha sido obtenida a partir de 
encuestas semiestructuradas. Esta forma de interactuación, con los habitantes de un territorio con el objetivo de conocer de primera mano las características del mismo, posibilita un nuevo modelo de innovación territorial que ofrece múltiples posibilidades al servicio del desarrollo socioeconómico del entorno, en el caso que no ocupa la gestión y conservación de especies vegetales con propiedades terapéuticas.

Palabras clave: Sierra de Mariola, participación ciudadana, biodiversidad, Sistemas de Información Geográfico.

\section{CITIZEN PARTICIPATION AS A TOOL FOR MANAGING PLANT BIODIVERSITY IN THE SERRA DE MARIOLA}

\section{AbSTRACT}

The Sierra de Mariola nature park has a great diversity of useful plants and a high ethnobotanical value, the proof is the existence of numerous studies and references linked to this territory made in the last two centuries.

Given the floristic richness of the area and the relationship between the natural environment and the population of the field of study, we understand that public participation is an essential tool for the management, conservation and enhancement of flora with therapeutical values in this natural protected area.

With this study we aim to highlight the importance of the knowledge that the society of Mariola has adquired throughout the years. The research process has paid out with participant observation, based on individualized consulting of people that are familiar with the most representative environmental floral species. All information obtained as a result of the processes of citizen participation has been channeled, managed and organized using Geographic Information Systems (GIS), thereby achieving a dynamic floristic inventory and consists of the most representative species with medicinal properties in Mariola. This information has been obtained from semi-structured interviews. This form of interaction with the inhabitants of a territory in order to see firsthand the characteristics, enables a new model of territorial innovation that offers many possibilities for socio-economic development of the environment, if they do not take the management and conservation of plants with therapeutic properties.

Key words: Sierra de Mariola, public participation, biodiversity, Geographic Information Systems

\section{INTRODUCCIÓN}

El parque natural de la Sierra de Mariola (DECRETO 3/2002, de 8 de enero, del Gobierno Valenciano, de declaración del Parque Natural de la Sierra de 
Mariola) nos ofrece múltiples posibilidades para el desarrollo de acciones que beneficien a su entorno socioterritorial, en el caso que nos ocupa presentamos un proyecto participativo, mediante el cual hemos elaborado un Sistema de Información Geográfica que nos permite diferenciar los diferentes usos medicinales de las especies vegetales de la Sierra de Mariola, de una manera intuitiva y visual. La flora terapéutica es sin duda una de las principales características de este espacio territorial estudiado en profundidad en los últimos siglos. En este sentido, los trabajos de Cavanilles, a finales del siglo XVIII, son decisivos para consolidar la idea de Mariola como un núcleo botánico de primer orden. Gracias a ello, a lo largo de todo el siglo XIX son muchos los botánicos extranjeros que, atraídos por la variedad y riqueza vegetal de la península Ibérica, incluyen la Sierra de Mariola en sus rutas de estudio. Webb (1826), Bourgeau (1852), Ross-Maessler (1854), Leresche (1862), Boissier y Reuter (1858), Hegel-Maier (1873 y 1878), Rouy (1880), Porta y Rigo (1885, 1890 y 1891), Dieck (1892) o Pau (1896), son algunos de los científicos de renombre internacional que recolectaron, estudiaron y describieron la flora de Mariola (Belda et al., 2004). Así, los numerosos estudios etnobotánicos existentes sobre la Sierra de Mariola: Font Quer (1995), Ferrer (2000), Pellicer (2000, 2001 y 2004), Ríos y Martínez (2003), Belda et al. (2004) y Belda y Bellod (2006), nos han servido de base para diseñar y ejecutar un proceso participativo, que nos ha permitido conocer de primera mano los conocimientos adquiridos de manera oral y mediante la observación de aquellos ciudadanos que han vivido o viven en permanente contacto con este espacio socioterritorial. Los participantes en el proceso son personas de un perfil concreto, caracterizado por tener costumbres rurales (agricultura, pastoreo, medicina tradicional, cazadores). Concretamente este estudio se centra en gente de avanzada edad que reside o ha residido en algún momento de su vida en el medio rural y que ha estado íntimamente relacionada con el mismo.

La identificación de los participantes se desarrolla a partir de la elaboración de un sociograma, esta técnica nos permite identificar de una manera clara los actores que van a participar en un proceso de participación ciudadana. En síntesis se trata de un gráfico en el cual se proyectan diferentes nodos que representan los actores existentes en el territorio así como las relaciones existentes entre ellos. Muestra "quién está en el proceso", "quién no está", "quién podría estar" y "quién impide que estén otros".

\section{Metodología: la Elaboración de un SIG DESDE la PARTICIPACión CIUDADANA}

La finalidad de un proceso participativo es la recopilación de información mediante la utilización de unas técnicas y métodos concretos. Para que un proceso participativo sea eficaz, los actores participantes deben tener acceso y conocer la información por igual, de lo contrario los actores participantes 
estarían en desigualdad a la hora de aportar contenido en el proceso. La información debe ser continuada y dosificada por parte del actor del proceso que la controla.

Según el manual de la diputación Provincial de Barcelona Eines per a la Participació ciutadana: bases, mètodes i tècniques, de la colecció Papers de Participació ciudadana, existen tres tipos de información que un proceso de participación ciudadana debe recoger o abordar:

- Información referencial: esta información apunta a la obtención de conocimientos objetivos sobre la temática abordada.

- Información reflexiva: profundizar en las diferentes visiones que se tienen desde distintas posiciones sociales. Se trata de identificar discursos compartidos, pero también avanzar en el desacuerdo, desde el intercambio y la reflexión colectiva.

- Información performativa: alimenta el proceso de tal manera que la comunidad desarrolle estrategias de acción colectiva en relación con los objetivos perseguidos.

Siguiendo estas premisas y con el objetivo de hacerlas efectivas elegimos las siguientes técnicas participativas para la obtención de información.

La entrevista en profundidad: es un método de recolección de información en el que hay un intercambio verbal entre un entrevistado y el entrevistador. Por tanto nos encontramos ante una técnica participativa en la que actúan dos actores: el entrevistador es el que recoge información mediante la elaboración de preguntas concretas y el entrevistado ofrece repuestas. (HAMMERSLEY, 1992). Con este método de investigación podemos obtener una información que con otros métodos sería imposible, ya que se recogen diversos significados, opiniones o experiencias del informante, además de gestos propios de una conversación que nos pueden servir de ayuda a la hora de analizar la información. La entrevista en profundidad se trata de un estadio más sofisticado y complejo de la conversación, el entrevistado debe de ser un actor con un amplio conocimiento del tema a tratar y por tanto el entrevistador debe conocer de antemano el tema a tratar para evitar pérdidas en la transferencia de la información. Existen tres tipos de entrevistas en profundidad, estructurada, semiestructurada y no estructurada. En este proyecto hemos utilizado las dos últimas.

Semi-estructurada: (HAMMERSLEY, M. 1992):

- El entrevistador posee una guía de entrevista, una serie de preguntas abiertas que realiza al entrevistado.

- Cada pregunta responde a una determinada cuestión del proyecto.

- Hay un orden pero sobre todo, la entrevista posee mayor flexibilidad.

- Como el entrevistador trata de contener y orientar la información que requiere del informante, suele ser intervencionista, aunque no en un grado tal como en las entrevistas estructuradas. 
No estructurada: (HAMMERSLEY, M. 1992):

- Existen diversos tipos: historia oral, historia de vida, experiencias etc.

- Más que en los contenidos, en la entrevista no estructurada la atención se centra en el informante.

- Se busca la capacidad del informante para expresar problemas, eventos o sucesos que conoce, con sus propias palabras.

- Es más impredecible y sobre todo, es el entrevistado quien lleva las riendas de la entrevista, no el investigador.

Encuestas: el primer paso que hay que realizar a la hora de elaborar una encuesta en un proceso participativo, es conocer de primera mano el tema que la motiva, para poder de este modo diseñar aquellas preguntas que nos den la información que precisamos. El segundo paso sería definir el universo al que nos hemos de dirigir, definiendo claramente la muestra de población sobre la que vamos a realizar el proyecto. Una vez conocido el contexto y definida la población a la cual va a ir dirigida la encuesta es el momento de diseñar el cuestionario. Este debe tener un formato amable y no debe abusar de términos tecnicistas, ya que lo que se pretende en un proceso de participación es que exista una buena interrelación entre los distintos actores del proceso (CEA, 1999).

Las preguntas a incluir en una encuesta deben ser aquellas que el técnico encargado del proceso de participación ciudadana considere que le van a ofrecer aquellas respuestas que considere básicas para la obtención de información. Es decir no existe un patrón de preguntas clave a la hora de realizar una encuesta pero en función del tipo de respuesta que acepten, las preguntas pueden adoptar alguna de estas formulas.

Con este trabajo participativo hemos recogido la base de información para la elaboración de un SIG que permite la localización, de una forma sencilla, de individuos o formaciones vegetales con un determinado uso popular. Así, se ha elaborado un sistema de información geográfica que incluye: género, familia, nombre científico, nombre popular en castellano y nombre popular en catalán y propiedades terapéticas de cada especie.

\section{Desarkollo del SiG}

La aplicación de una herramienta como los Sistemas de Información Geográfica en un trabajo de este tipo sin duda proporciona numerosas ventajas. Los SIG contienen una base de datos georreferenciada y una serie de mecanismos que, conjuntamente, nos permiten adquirir, almacenar, analizar y representar información geográfica (espacial y temática) (LARROSA, 2004). Pero lo que diferencia a los SIG de otros programas afines, como son los de cartografía asistida por ordenador o los gestores de bases de datos, es su capacidad para almacenar de manera interdependiente e interactiva los datos espaciales (por ejemplo la ubicación de una planta concreta) y su componente temática (usos 
medicinales) (Larrosa, 2004). Esa capacidad hace de los SIG un instrumento potente de gestión y análisis de cualquier variable territorial. Los Sistemas de Información Geográfica nos han permitido ubicar de forma rápida y sencilla los taxones estudiados, al mismo tiempo que nos ha servido como potente herramienta cartográfica para su representación.

Gestión de la información: una vez identificadas las especies citadas por los informantes, siguiendo la metodología Crespo y Mateo (2003), se procede al marcaje georreferenciado de individuos de cada una de las especies citadas.

Este es el proceso más minucioso y a la vez apasionante a la hora de elaborar un estudio de localización de taxones, ya que implica realizar trabajo decampo. Así, la marcación de los distintos taxones mediante el empleo del GPS de alta precisión (Trimble®) se realizó en varias jornadas de campo, localizando siempre que fuera posible cinco individuos de cada una de las especies analizadas en el estudio.

El manejo del GPS ha permitido dar un nuevo impulso a las cartografías de localización, ya que ha facilitado con creces las labores de campo. El trabajo de campo se completa en el gabinete con la corrección y exportación de datos a la base de datos. La corrección diferencial de los puntos marcados la hemos llevado a cabo utilizando el software GPS Phatfinder®. Una vez corregidos los puntos, el siguiente paso es la construcción de una capa *.shp con el software Arcgis ${ }^{\circledR}$, para que queden registrados todos los taxones y de este modo poder tratar los datos cartográficamente según nos convenga. De este modo, se han realizado los diferentes mapas de localización, empleando la herramienta layout de dicho software.

Al quedar toda esta información registrada en una base de datos vinculada a un espacio territorial concreto, la aplicación nos resultará de gran utilidad a la hora de identificar individuos y sus distintas propiedades medicinales.

Elaboración de la base de datos: la creación de la base de datos es el elemento fundamental del proyecto, ya que nos permite tener un registro de taxones amplio y modificable en cualquier momento. De esta forma, la elaboración de esta base de datos se ha realizado en dos fases: la primera antes de los trabajos de campo y la segunda después. Así, en la primera fase, tomando como referencia las entrevistas entobotánicas se introdujeron los datos referidos a género, familia, nombre científico, nombre popular en castellano, nombre popular en valenciano y usos terapéuticosde cada una de las especies. En la segunda fase, se introdujeron las coordenadas (x,y) de cada individuo localizado en el campo. Así, una vez conocidas y registradas las coordenadas en la base de datos el paso último es la creación de la cartografía dependiendo de las necesidades de cada momento. 


\section{Potencial para el Desarkollo local}

La aplicación de la participación ciudadana combinada con los Sistemas de Información Geográfica proporciona numerosas ventajas, algunas ya mencionadas a lo largo del trabajo. Los SIG contienen una base de datos georreferenciada y una serie de mecanismos que, conjuntamente, nos permiten adquirir, almacenar, analizar y representar información geográfica (espacial y temática). Pero lo que diferencia a los SIG de otros programas afines, como son los de cartografía asistida por ordenador o los gestores de bases de datos, es su capacidad para almacenar de manera interdependiente e interactiva los datos espaciales (por ejemplo la ubicación de una planta concreta) y sus propiedades medicinales. Esa capacidad hace de los SIG un instrumento potente de gestión y análisis de cualquier variable territorial. Los Sistemas de Información Geográfica nos han permitido ubicar de forma rápida y sencilla los taxones estudiados, al mismo tiempo que nos ha servido como potente herramienta cartográfica para su representación. Además, nos hemos valido de las potencialidades de los SIG en el ámbito de las búsquedas para seleccionar los distintos taxones estudiados en función de los usos ATC requeridos. De la misma manera, podríamos realizar, una vez finalizado el trabajo de campo y la agregación de variables en las bases de datos correspondientes, multitud de consultas de tipo espacial y temática, así como operaciones más complejas de análisis espacial y de vecindad, cuestiones que se podrían plantear en trabajos futuros, siempre contando con la involucración social.

La información recopilada en este estudio puede permitir la puesta en valor de estos recursos naturales de una forma muy dinámica y productiva. Así, la utilización de elementos vegetales con diferentes usos puede ser útil en el caso de empresas dedicadas a la producción de infusiones, extracción de esencias, remedios naturales, colorantes, extracción de fibras y alimentos. De este modo, se puede promover el cultivo de estas especies para promover su explotación y minimizar su extracción del medio natural y al mismo tiempo, promover el empleo local. Por otro lado, este tipo de actividades son de gran interés para promocionar el turismo local, ya que los clientes cada vez más reclaman este tipo de recursos. Además, la explotación apícola asociada a esta diversidad de especies botánicas es clave para mantener los sistemas mediterráneos y potenciar la polinización y a su vez, la producción de frutos.

\section{REsultados}

Se recogieron 93 especies de plantas, pertenecientes a 41 familias botánicas, que se utilizan para diferentes propósitos. Se registran los nombres científicos de las especies de plantas, el pliego de herbario depositado en el Departamento de Ecología de la Universidad de Alicante, la familia a la que pertenecen, la frecuencia de citación por los informantes, importancia cultural, si se trata de 
especies silvestres o cultivadas, y sus propiedades terapéuticas para los seres humanos. De este modo, 464 individuos fueron localizados del total de 93 especies en el área de estudio. Se han construido 37 mapas para cada uno de los diferentes usos identificados.

Con respecto a la recolección de las diferentes plantas útiles, la mayoría se recogen en matorrales o bosques $(45,16 \%)$, otras se asocian a las viviendas rurales, senderos, huertos familiares, etc.; el $12.90 \%$ a las zonas urbanas o periurbanas, el 9,68\% de las especies se recogen de las áreas cultivadas, el 5.38\% en lugares rocosos y el 3.23\% se obtienen de ambientes acuáticos. La mejor temporada para la recolección de plantas silvestres es la primavera, cuando la mayoría de las especies están disponibles.

Labiatae $(n=19)$, Compositae $(n=9)$ y Leguminoseae $(n=6)$ son las familias más representadas entre las plantas que se han utilizado para diferentes propósitos en humanos. En este estudio, la mayoría de las especies son silvestres $(n=66)$ y las otras se cultivan $(n=27)$ en las tierras de cultivo y casas de campo. Las especies vegetales más importantes utilizadas por los informantes son Foeniculum vulgare, Chiliadenus saxatilis, Sideritis angustifolia, Salvia blancoana, Rosmarinus officinalis y Thymus vulgaris, que representan más del $80 \%$ de la frecuencia de citación relativa (RFC).

BIBLIOGRAFÍA

BELDA, A. y BELLOD, F.J. \& RIOS, S. (XII-2004): Avance sobre la flora medicinal en la Sierra de Mariola. Flora Montiberica vol. 28, pp. 29-48.

BELDA, A. y BELLOD, F.J. (2006): Plantas medicinales de la Sierra de Mariola. Servicio de Publicaciones de la Universidad de Alicante. 294 pp. BELDA, A y Otros (2009): Análisis de la biodiversidad de fauna vertebrada en el Parque Natural de la Sierra de Mariola mediante fototrampeo. Mediterránea Serie de Estudios Biológicos no 20: 7-34.

CEA D'ANCORA, M.A. (1999): Metodología cuantitativa. Estrategias y técnicas de investigación social. Madrid: Sintesis.

CRESPO, M.B. \& MATEO, G. (2003): Manual de determinación de la flora valenciana, Monografías de flora Montibérica. $501 \mathrm{pp}$.

HAMMERSLEY, M. (1992): "Deconstructing the qualitativequantitative divide" en J. Brannen (editor), Mixing qualitative and quantitative research, Avebury, Aldershot, USA.

LARROSA ROCAMORA, J.A. (2004): "Sistemas de Información Geográfica en Geografía Humana", en Segrelles Serrano (coord.): Geografía Humana. Fundamentos, métodos y conceptos, Editorial ECU, Alicante, 191-222 pp. PELLICER, J. (2000): Costumari botanic vol. 1 edicions Bullent, Valencia. 252 pp. PELLICER, J. (2001): Costumari botanic vol. 2 edicions Bullent, Valencia. 252 pp. PELLICER, J. (2004): Costumari botanic vol. 3 edicions Bullent, Valencia. 257 pp. 